\title{
A Governance Framework for Adopting Agile Methodologies
}

\author{
Carine Khalil $^{1 *}$, Sabine Khalil ${ }^{2}$ \\ 1 Paris Descartes University, 45 Rue Des Saints-Pères, Paris, France. \\ 2 Telecom Paris Tech, 46 Rue Barrault, Paris, 75013, France. \\ * Corresponding author. Tel.: 0646734436; email: carine.khalil@parisdescartes.fr \\ Manuscript submitted October 9, 2015; accepted February 19, 2016. \\ doi: 10.17706/ijeeee.2016.6.2.111-119
}

\begin{abstract}
Even though the number of agile adopters in software organizations keeps on growing, many challenges are still encountered during the integration of agile practices across software projects. Only few succeed in becoming agile especially when the organizational context is not aligned with agile philosophy and principles. There is an evident need for providing organizations with a governance framework that helps them address the decision-making environment throughout the adoption process of agile methodologies. This paper proposes a governance framework based on the IT governance model of Weill (2004). By defining agile decision domains as well as agile decision input and rights, the framework aims at assisting organizations in selecting the appropriate agile practices and aligning them with business objectives, project team needs in addition to the organizational infrastructure.
\end{abstract}

Key words: Agile adoption, agile key decisions, agile decision input and rights, IT governance framework.

\section{Introduction}

Software firms operate in a competitive market and in a dynamic environment. These firms need to deliver, on time, reliable and flexible solutions that meet customers' evolving needs. Over the last two decades, software teams have progressively adopted new managerial principles and tools to help them manage and integrate changes in complex software solutions. As a result, a set of new "methodologies" known under "agile" methodologies has emerged and increasingly gained popularity among software practitioners (VersionOne ${ }^{1} \mid$ National Survey $^{2}$ ). The managerial principles, on which these methodologies rely, question the "conventional" project management approaches based on up-front requirements identification, extensive documentation, and sequential development. These approaches, such as the "V" and the "waterfall" lifecycles, are perceived as being unable to cope with the inevitable changing demands and rapid technological innovations [1], [2].

Agile methodologies emphasize short iterative development cycles (from one to three weeks), close collaboration between the developers and the client, as well as, adaptation to continuous changes. Each iteration consists of delivering an incremental version of the product that can be tested by the client. Therefore, customer feedback is encouraged and can be integrated in the next version of the product. Unlike conventional approaches, requirements are defined iteratively based on customer prioritization. Even

\footnotetext{
${ }^{1}$ https://www.versionone.com/pdf/2013-state-of-agile-survey.pdf

${ }^{2} \mathrm{http}: / /$ fr.slideshare.net/xwarzee/enqute-2011-vous-votre-organisation-et-agile
} 
though agile methodologies seem to improve the sharing vision of the project team and enhance customer satisfaction as well as product quality [2], [3], the adoption of these methodologies remains a challenge for many large organizations and software teams. Only few succeed in becoming agile especially when the organizational context is difficult to align with agile philosophy and principles. Many research papers have tried to address the challenges faced in distributed and complex software environments [4]-[6]. Yet, recommendations have either treated a small aspect of the development process or focused on a single organization with very specific characteristics. As a result, the integration of agile practices has rarely succeeded at the global level. In this respect, it seems critical to provide organizations with a guideline that helps them determine which agile practices they should implement, in addition to who should be involved in the decision-making process during the adoption of these practices. To our knowledge, no governance model exists for assisting large organizations in adopting agile methodologies. Accordingly, this research paper aims at proposing a framework that guides large and complex ${ }^{3}$ organizations in adopting agile practices and aligning them with their business strategies, project team needs, and organizational infrastructure.

We drew on Weill and Ross's IT governance model in order to address our research goal. IT governance is about specifying the framework for decision rights and accountabilities to encourage appropriate behavior in the use of IT [7]. This behavior should be consistent with the organization's goals, structure, and culture. Hence, while the existing literature on IT governance appears to be relevant to the problem of agile adoption, the lack of governance can be viewed as a disablement to agile transformation.

This paper is structured as follow: Section 2 will review the related work of agile software development. Section 3 will introduce the IT governance model. Based on [7], Section 4 will propose an agile adoption governance framework. This framework defines agile key decisions as well as archetypes that can be encountered within organizations that want to become agile. Finally, Section 5 will discuss the suggested framework and the concluding remarks of this research work.

\section{Agile Methodologies}

Different agile methods exist. However, these methods are based on common values and principles written in the Agile Manifesto ${ }^{4}$, where each emphasizes a set of practices and tools. It is beyond the scope of this paper to explore each of the practices and tools. In general, these methodologies emphasize iterative development, self-organizing teams, code quality through continuous integration, unit-testing, feedback, as well as, adaptation to changes. Therefore, the delivery of an incremental working product is valued over excessive documentation and up-front planning. Furthermore, constant interactions as well as collaboration practices such as daily meetings, retrospectives, client-on-site, pair programming, and more, are encouraged over formal processes and tools such as weekly reports, documentation deliverables, etc.

Yet, agile transition remains an unpredictable process and a challenge for many organizations. Many factors seem to affect the integration of agile practices, particularly, within large and complex environments. Beyond geographical distribution and large-scale projects, other contextual factors such as team composition, project manager authority, organizational culture, as well as existing management systems seem to constrain the integration of agile practices [8], [9]. Actors collectively develop and organize the adoption processes of agile methodologies without, however, relying on a structured approach. Through constant interactions and negotiations, they make sense of these methodologies as well as their implementation context, and try to adapt them to their specific situations [10]. Nevertheless, the organizing

${ }^{3} \mathrm{~A}$ complex organization refers to an organization characterized with a matrix structure, large projects and unstable project teams.

${ }^{4} \mathrm{http}: / /$ www.agilemanifesto.org 
processes become very challenging when the organizational context is complex and problematic. For instance, close collaboration and mutual adjustments become difficult to achieve in distributed and large organizations [4]-[6]. People in charge of implementing agile practices in such environments face many challenges on the organizational, managerial, cultural and human level [8]. Distributed development team members can be reticent regarding the introduction of collaboration practices, including daily meetings, pair programming, client-on-site, etc. Furthermore, the cooperation between agile developers and "traditional" project teams can be very challenging. Combining up-front planning and iterative development requires a lot of efforts in terms of synchronization and coordination between different team members [11]. Hence, large and complex organizations struggle to embrace agile principles and tools, especially when they encompass plan-driven projects. Often, people involved in the agile transformation process are not capable of identifying the right agile practices that can fit their organization, accommodate the contextual characteristics, and respond to their business needs. Therefore, good governance seems essential for clarifying who has accountable input rights for the agile transformation process. Previous research studies have rarely focused on the impact of decision input and rights in the adoption process of agile practices. Yet, the decision-making process seems to play an important role in the adoption of these methodologies. Therefore, managers should discuss which decisions need to be made while adopting agile practices, and who should have input and rights for each of these decisions.

\section{IT Governance Model}

IT governance serves many objectives, including allocating the right people to the right decisions and maximizing value creation through aligning business with IT. [7] developed an IT governance framework that includes three major components: domain (what decisions need to be made?), style (who has input/ decision rights?) and mechanism (how are the decisions formed and enacted?). In this research paper, we will only focus on the first two components: domain (what decisions need to be made?) and style (who has input/ decision rights?). Even if mechanism is also a fundamental component in IT governance, it is beyond the scope of this paper to address the way each decision is aligned and communicated within software organizations that intend to adopt agile methodologies.

\subsection{Domains - The Five Key Decisions}

Organizations, especially large ones, deal with five major IT decisions in order to define the IT scope: IT principles, IT architecture, IT infrastructure strategies, business application needs, and IT investment and prioritization. IT principles are defined as simple statements representing an organization's beliefs regarding the way IT is used on the long term. Enterprises need to decide which business principles will dictate the role of IT in the business. In fact, the short list of business principles that define the enterprise's business strategy will lead to a set of IT principles. These principles are used as a communication bridge between technical experts and top managers. IT architecture discusses the way the core business processes (product development, product/service delivery, employee development and satisfaction, technology management, quality, etc.) are implemented in IT. IT architecture is also referred to as a set of detail standards and policies responsible for the design of infrastructure technologies, applications and databases leading to the way business will be done [12]. It is the integrated set of technical choices that guide the organization in satisfying business needs. IT infrastructure refers to a set of reliable and centrally coordinated strategies constituted by human and technical IT capabilities (computer, database software, operating systems, knowledge, skills, standards). It provides the foundation for the enterprise's IT capability and aims at monitoring, controlling and supporting IT services. The fourth major IT decision is related to the business application needs, where these needs are satisfied by purchased or internally developed IT applications. Business applications have to respond to two conflicting goals: creativity (finding 
new ways to deliver customer value) and discipline (ensuring that applications leverage the existing enterprise architecture). Finally, the last major IT decision is regarding the IT investment and prioritization, which concerns the amount and the place to invest in IT. The business decision makers should decide which business processes will receive IT support and which will not.

\subsection{Styles - The Input and/or Decision Rights}

Once the scope of IT has been defined, organizations need to allocate each decision to a proper group. Ref. [7] proposed that an organization should identify who contributes to the decision making process (who has input), and who makes the decision (who has the decision rights). After analyzing 256 companies in 23 countries, [7] found out that IT decision are mostly held by one of these six groups: business monarchy, IT monarchy, IT duopoly, feudal, federal, or anarchy. Business monarchy regroups a set of senior business executives and managers (CEO, CIO, COO, etc.). IT monarchy is a group of IT executives/directors responsible for making different decisions. In IT duopoly archetype, decisions come from an agreement between IT executives and a business group. This archetype is divided between an IT and a business representation. The feudal archetype consists of having each business unit make their own decisions in order to optimize their local needs. However, this archetype is barely found in organizations since synergies across business units are primordial for them. The federal archetype attempts to balance multiple governing bodies working on different hierarchical levels of business (business unit leaders, key process owners, or their delegates). The last archetype is anarchy. It is close to the feudal archetype where each group makes their own decisions based on their needs. The difference is that anarchists speak for small groups while feudals represent larger groups.

\section{Designing a Governance Framework for Agile Adoption}

Each software organization is a unique combination of actors, management practices, organizational structure, policies, values, norms, and so on. Therefore, every single organization will experience the adoption process of agile methodologies differently, even though each methodology clearly defines the practices, roles, and tools that software teams are supposed to adopt. The adoption of agile practices can be viewed as an organizing process based on constant interactions between actors that are making sense of these methodologies and trying to reach a consensus regarding their implementation [8]. From this perspective, it seems essential to provide organizations with a governance framework that helps them address the decision-making environment throughout the adoption process of agile methodologies. By drawing on [7], this research paper proposes a model that assists organizations in the decision-making processes during the integration of agile methodologies.

\subsection{Agile Key Decisions}

As a first step, organizations involved in the adoption process of agile practices have to delimitate the scope of the agile methodology they want to implement. To do so, they have to cope with five key decisions that enable them to translate their business principles and project team needs into agile principles and practices. These decisions should cover the following domains: agile principles, agile architecture, agile infrastructure, project team needs, and agile project investment and prioritization.

Agile principles consist of high-level decisions about the strategic impact of agile practices in the business In this respect, organizations should determine their desired operating model and prioritize their business principles in order to guide the adoption of agile principles. In fact, agile principles can shape business principles by becoming more client-oriented or quality-oriented, for example. At this stage, questions regarding the role of agile practices in the organization, the desirable agile behaviors, the funding to the agile adoption projects, and many others, can be addressed while discussing the agile principles. Project 
team needs invoke team members' priorities regarding software project requirements. In order to stay competitive, software project teams must find new ways to deliver customer value creativity in order to meet business needs. Thus, they need to identify wastes and dysfunctions encountered in their development process in order to address them through a set of best practices. Non added value activities such as unused documentation or functionalities, code defects or unoccupied actors waiting for information can increase project costs while reducing team's performance and product quality. In this respect, agile practices and tools are selected to respond to these problems. Questions that should emerge are about which business opportunities could be created through new management techniques, the type of problems the project team usually reports, the way project wastes can be addressed, the way the development process can be improved or even the way agile project outcomes can be assessed. Agile architecture refers to the integrated set of agile technical choices that will guide the organization and project teams in satisfying their needs. At this level, agile practices and tools are selected in order to respond to business needs and project teams' priorities. Project managers should be wondering about the core projects of the organization and how they are related. They can also conjecture which processes and people should be involved in the projects, and the way information must be shared across projects. In addition, they should think about the choices regarding the different agile practices that should be implemented, as well as, the technologies that will support their implementation. Agile infrastructure is the foundation for the enterprise's agility. It consists of establishing an alignment between agile practices and the organizational structure and culture. Regarding agile infrastructure, project managers should examine the way the organizational structure can support the implemented agile practices (new responsibilities, roles, communication channels, etc.) and the way agile practices fit the organizational infrastructure. Beside, it is vital to implement collaboration tools, involve suitable people and appropriately define their roles and responsibilities. In addition, these people should attend training programs to increase their knowledge and effectiveness in an agile environment. Agile project investment and prioritization refers to investment decisions regarding agile projects. Organizations should decide how much and where to invest in agile projects. In addition, they should choose the most strategic projects for the organization and the ones that are ready for agility. Table 1 summarizes the addressed questions regarding the different agile decisions.

Table 1. The Addressed Question in Agile Key Decisions

\begin{tabular}{ll}
\hline Agile Decisions & \multicolumn{1}{c}{ Addressed Questions } \\
\hline \multirow{3}{*}{ Agile Principles } & What is the role of agile principles in the business? \\
& What are the agile desirable behaviors? \\
& How the agile adoption project will be funded? \\
Project Team Needs & What business opportunities are created via new management practices? \\
& How can project wastes be addressed? \\
& What are the project risks? \\
Agile Architecture & How information is shared across projects? \\
& What agile practices must be implemented? \\
& What technology choices will support agile practices? \\
Agile Infrastructure & What technical guidelines and standards must be used? \\
Agile Project Investment and & How organizational structure can support agile practices \\
Prioritization & How to enable collaboration between team members \\
\hline
\end{tabular}

\subsection{Agile Decision Input and Rights}

The second step in designing agile adoption governance is to determine who should make decisions and 
provide input for each key decision. Ref. [7] identified six governance archetypes for making IT decisions. These archetypes refer to combinations of people who have either decision rights or input rights to IT decisions. Applied to agile adoption process, these archetypes enable us to understand how decisions rights and input can be made within software environments and how they can affect the adoption process of agile methodologies. In fact, people involved in agile decision rights and/or input have a critical role in the adoption process. In this respect, key stakeholders should be analyzed and identified.

In order to better define each archetype, we drew on stakeholder analysis literature in project management and identified the groups of people that are usually involved in software project management decisions [13], [14].

Business monarchy includes senior business executives, business leaders, financial chief officer, and senior leadership teams. Business monarchy concerns the highest hierarchical level at the organization, more specifically, the executives level. Business monarchies are usually responsible for taking project investment and prioritization decisions. Agile monarchy consists of agile executive groups (support the agile initiative and play a proactive role in communicating and managing expectations with other executives), agile leader groups (middle management in charge of leading the agile initiative and providing support to foster engagement, alignment and adoption across all levels of the organization), agile development teams, product owner group (group of product managers working together to adopt new product management and development practices in a more agile way), or other agile professionals that take decisions regarding agile architecture and infrastructure. However, it is still very rare to find agile executive groups within organizations. The feudal model refers to business unit leaders, business managers, customer representatives, project managers, functional managers as well as business process owners. The federal decision making model attempts to balance responsibilities and accountabilities of multiple governing bodies such as agile representatives, business leaders/executives, business owners, business units, functional managers, project managers as well as customer representatives. Agile duopoly is a two party arrangement where decisions result from agreements between agile representatives and business groups (typically CxOs) or between agile representatives and business units, functional managers or process owners. And finally, in an anarchy archetype, individuals or small groups make their own decisions based on their own needs. This archetype can be found within small and self-organizing software teams. Anarchies are supported where local customers require very rapid software development solutions.

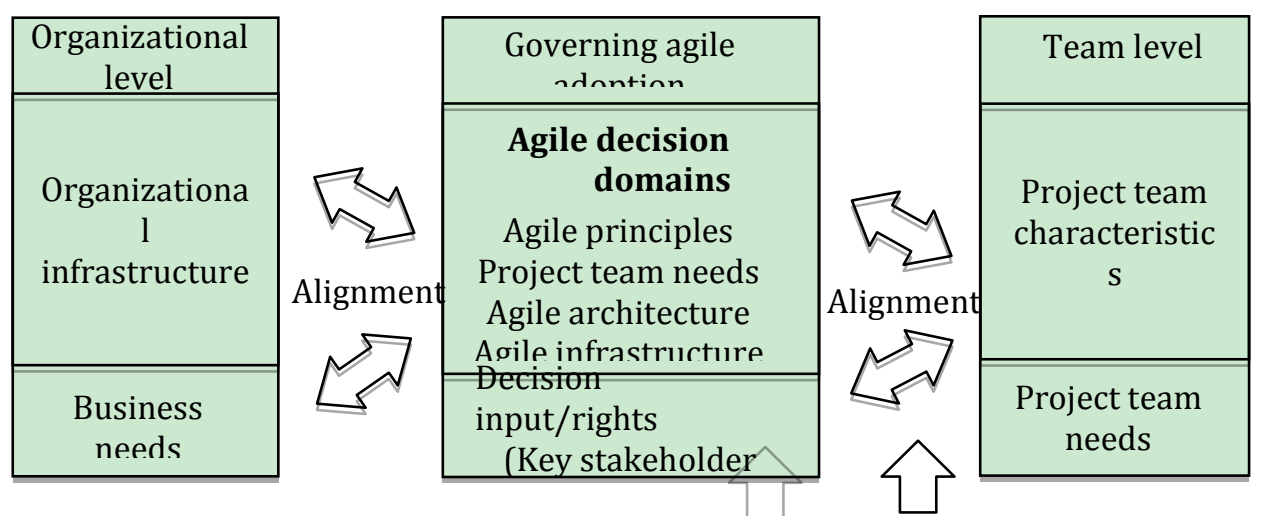

Fig. 1. Agile adoption governance framework.

No survey has been conducted in order to examine decision rights and input during the agile adoption process. In addition, previous research work on agile implementation has rarely focused on the impact of decision input and rights in the adoption process of these practices. Yet, decision-making process seems to play an important role in the adoption of agile methodologies. In [8], technical decision input (agile 
architecture and infrastructure) was provided somehow by the wrong people, which has negatively affected the implementation process. The choice of agile practices didn't fit the organizational infrastructure and project team needs [8]. In fact, the lack of participation of agile representatives in decision input and rights can be considered as one of the failure factors of agile implementation within the organization. Additionally, decisions rights should combine different groups and not be limited to one group such as business executives or business unit leaders. People involved in the adoption process of agile practices must be aware of agile related risks and their impact on the organization. Thus, it is important to involve the right people in providing input and making decisions. In this regard, the federal model that involves multiple governing bodies in the decision-making of agile adoption process may help organizations in the implementation of agile methodologies. Accordingly, different hierarchical levels of business as well as technical services will collectively participate in aligning agile principles with business goals and project team needs. Moreover, consultants such as experts in the agile domain can provide useful input for these decisions. On one side, they assist business leaders in clarifying their strategy as well as prioritizing their objectives and goals, whereas on the other side, they help project teams in mapping the encountered wastes throughout the project and defining agile principles that can address these difficulties and respond to business principles.

\section{Discussion and Conclusion}

Even though the number of agile adopters in software organizations keeps on growing, many challenges are still encountered during the integration of agile practices across software projects. In fact, research studies on agile adoption have mainly focused on challenges faced by software developers directly involved in the development process of the product. The integration of agile practices at the global level of the organization has rarely been addressed. Up till now, no structured approach exists to guide large organizations throughout the adoption of agile practices especially that these practices promote organizing activities as well as mutual adjustments between project teams. In this regard, structuring their use within organizations is difficult to achieve. It would be more judicious to structure their adoption by proposing a governance framework on which organizations, that are seeking agility, can rely.

This agile governance framework stresses on five key decisions that organizations need to take into consideration when adopting agile methodologies. It also portrays the groups of people that can be involved in agile decision input and rights. By addressing these decisions and identifying who should be held responsible for them, organizations can better align agile methodologies with their organizational infrastructure (culture, structure, roles), business goals as well as project team needs. Therefore, the involvement of the right people in decision input and rights is essential for succeeding the adoption process.

This research paper offers practical and theoretical contributions in agile software development research field. On the one side, this paper provides an agile adoption framework that direct and assists software practitioners, consultants, business groups, leading change committees, etc. in identifying what business-oriented and technical-oriented decisions need to be made and by who. As previous research has shown, transitioning to agile is not a simple process. Agile methods, as described by their authors, need to be adapted to each organizational and managerial context. In this respect, by relying on a governance framework for adopting agile methodologies, organizational actors become aware of agile decisions that need to be addressed in order to determine what agile initiatives better meet their organizational infrastructure, business goals and project team needs. However, this framework has not been experimented yet which can be viewed as a limitation of this paper. However, our next research objective is to test this framework on a large panel of software organizations. Data findings will help us adjusting the current governance framework and evolving its components. On the other side, this paper contributes to the 
existing literature on agile methodologies. It offers a new lens for addressing the adoption process of these lightweight methodologies.

The fact that we didn't address decision mechanisms can be viewed as another limitation of this study. However it is more legitimate to examine the way decisions are formed and enacted before integrating the appropriate behaviors in the framework.

\section{References}

[1] Poppendieck, M., \& Poppendieck, T. (2007). Implementing Lean Software Development: From Concept to Cash. Pearson Education.

[2] Petersen, K., \& Wohlin, C. (2009). A comparison of issues and advantages in agile and incremental development between state of the art and an industrial case. The Journal of Systems and Software, 82(9), 1479-1490.

[3] Karlström, D., \& Runeson, P. (2005). Combining agile methods with stage-gate project management. IEEE Software, 22(3), 43-49.

[4] Paasivaara, M., Durasiewicz, S., \& Lassenius, C. (2008). Distributed agile development: Using Scrum in a Large Project. Global Software Engineering, 87-95.

[5] Viktorov, A., Blount, J., \& Puntikov, N. (2007). Distributed scrum: Agile project management with outsourced development teams. Proceedings of 40th Annual Hawaii International Conference on System Sciences (pp. 274-274).

[6] Kircher, M., Jain, P., Corsaro, A., \& Levine, D. (2001). Distributed extreme programming. Proceedings of the $2^{\text {nd }}$ Conference on Extreme programming and Flexible Processes in Software Engineering.

[7] Weill, P., \& Ross, J. W. (2004). IT Governance on One Page (CISR WP No. 349 and Sloan No. 4526-04). Massachusetts Institute of Technology.

[8] Khalil, C. (2011). Les méthodes "agiles" de management de projets informatiques: Une analyse "par la pratique. Doctorat thesis in management sciences, LTCI, Telecom ParisTech, Paris.

[9] Khalil, C., \& Fernandez, V. (2013). Agile management practices in a "lightweight" organization: A case study analysis. Journal of Modern Project Management, 1(1).

[10] Khalil, C., \& Fernandez, V. (2011). The implementation of agile project management: A practice-based analysis. Proceedings of the 27th Egos colloquium. Gothenburg, Sweden.

[11] Boehm, B., \& Turner, R. (2005). Management challenges to implementing agile processes in traditional development organizations. IEEE Software, 22(5), 30-39.

[12] Ross, J. Creating a strategic IT architecture competency: Learning in stages. MIS Quarterly Executives, 2(1), 2003.

[13] Anderson, J. (2013). The Lean Change Method: Managing Agile Organizational Transformation Using Kanban, Kotter, and Lean Startup Thinking.

[14] Project Management Institute (PMI). (2014). Pulse of the profession in-depth report: Executive sponsor engagement-Top driver of project and program success. Retrieved from the website: www.pmi.org/ /media/PDF/Knowledge\%20Center/PMI-Pulse-Executive-Sponsor-Engagement.ashx

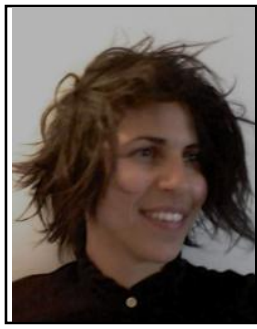

Carine Khalil was born in Beirut-Lebanon in 1982. She has a PhD in information system management delivered by Telecom ParisTech, Paris-France in 2011.

She also has a master degree in organization management delivered by IAE-Sorbonne, Paris in 2007 and a master degree in human resources management delivered by Saint-Joseph University-Beirut in 2006.

She is currently a professor and researcher at Paris Descartes University since 2013. 
She conducts research at the Interdisciplinary Laboratory for Applied Research in Health Economics (LIRAES-Paris Descartes). She is specialized in information systems and management, agile software development and project management methodologies. She is also certified scrum master from Scruminc. Before 2013, she was a postdoctoral researcher at Ecole Centrale Paris, France. During her postdoctoral fellowship, she conducted two research projects on Knowledge Management and Information Systems and published a paper in the journal "Système d'Information et Management".

She has been involved in numerous research projects with different international groups (Orange Telecommunication Group-Paris, SNCF-Paris, ETOP International Group) that were interested in implementing information systems and innovative management practices. She's the author of the book "Les méthodes agiles en développement informatique" edited by Presse des Mines-Paris in 2013.

Dr. Carine Khalil published many papers related to the implementation of agile methodologies within large and complex organizations. Among these papers: "Can agile collaboration practices enhance knowledge creation between cross-functional teams?, Springer verlag; « Entre autonomie et contrôle : quelle régulation pour les systèmes de gestion des connaissances? », Système d'Information et Management and "Agile management practices in a "lightweight" organization: a case study analysis", Journal of Modern Project Management.

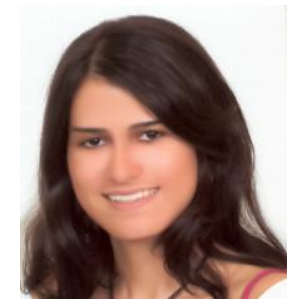

Sabine Khalil was born in Lebanon in 1990. She received her bachelor degree in electrical and computer engineering from the American University of Beirut, Lebanon in 2012. Then, she received her master's degree in engineering management from the American University of Beirut, Lebanon in 2014. In November 2014, she joined Telecom ParisTech, Paris for a PhD on the management of cloud computing in organizations. Ms. Khalil's research interests are cloud computing, information technology, governance, and organizational restructuring. 